\title{
AVALIAÇÃO DO POTENCIAL REPRODUTIVO DE TOUROS DA RAÇA BLONEL
}

Ana Paula Balduíno Rabelo, Eduarda Gonçalves Damacena, Vitor Lemes da Silva, Wallacy Barbacena Rosa dos Santos, Andreia Santos Cezário, Jefferson Corrêa Ribeiro, Eliandra Maria Bianchini Oliveira, Aline Souza Camargos

Departamento de Zootecnia - Instituto Federal de Educação, Ciência e Tecnologia Goiano - Campus Morrinhos Morrinhos, GO. E-mail: anapaulabr73@hotmail.com

\section{RESUMO}

A busca dos bovinos de corte ideais para países tropicais, como o Brasil, elevou a raça Blonel (5/8 Blonde e 3/8 Nelore). Esta é a mais nova raça de bovinos registrados pelo Ministério da Agricultura, Pecuária e Abastecimento (MAPA). Este trabalho teve por objetivo avaliar o desempenho reprodutivo de touros da raça Blonel, por meio de análise mediata e imediata de parâmetros do ejaculado. Especificamente, avaliar a análise imediata e mediata de parâmetros do ejaculado. A coleta de dados reprodutivos foi feita no ano de 2012, no rebanho da Fazenda Três Furnas em Buriti Alegre - GO. Foram estudados 14 animais submetidos a exame andrológico entre os 24 e 63 meses de idade. $O$ exame andrológico foi realizado pelo mesmo veterinário, durante os meses de agosto e setembro. Imediatamente após a coleta, foram realizadas análises imediatas do sêmen: motilidade, vigor e concentração de esperma. Os dados dos relatórios foram coletados, digitados em planilha, sujeitos a análise e cálculo médio. Para análise estatística, os resultados foram submetidos a ANOVA a $5 \%$ do nível de significância. Os resultados (média \pm desvio padrão) foram: $30,14 \pm 1,34$ e $28,93 \pm 1,69 \mathrm{~cm}$ para a circunferência escrotal, 5,5 $\pm 0,0$ e 4,64 $\pm 0,8 \mathrm{ml}$ para volume de sêmen, $2,71 \pm 0,49$ e $2,57 \pm 0,53 \%$ para o turbilhonamento, $54,28 \pm 6,07$ e $67,86 \pm$ 9,51\% para motilidade, 3,57 $\pm 0,79$ e 3,28 $\pm 0,75 \%$ para vigor, $657,14 \pm 97,59$ e $678,57 \pm 138,01 \%$ para concentração, $7,57 \pm 1,27$ e 5,71 $\pm 2,43 \%$ para defeitos maiores totais, $5,43 \pm 2,76$ e 2,29 \pm $1,60 \%$ para defeitos menores totais para touros jovens e adultos, respectivamente. Os resultados observados neste estudo estão dentro dos parâmetros aceitáveis para bovinos dessas faixas etárias.

Palavras-chave: bovino; concentração espermática; motilidade; sêmen; volume

\section{EVALUATION OF REPRODUCTIVE POTENTIAL OF BULLS OF BLONEL RACE}

\begin{abstract}
The search for beef cattle ideal for tropical countries, such as Brazil, raised the Blonel breed (5/8 Blonde and $3 / 8$ Nellore). This is the newest breed of cattle registered by the Ministry of Agriculture, Livestock and Supply (MAPA). The objective of this study was to evaluate the reproductive performance of bulls of the Blonel evaluating the immediate and immediate analysis of ejaculate parameters. Specifically, evaluate the immediate and mediate analysis of ejaculate parameters. The collection of reproductive data was made in the year 2012, in the herd of Fazenda Três Furnas in Buriti Alegre - GO. We studied 14 animals submitted to andrological examination between 24 and 63 months of age. The andrological exam was performed by the same veterinarian, during the months of August and September. Immediately after collection, immediate semen analyzes were performed: motility, vigor and sperm concentration. The data of the reports were collected, typed in spreadsheet, subject to analysis and average calculation. For statistical analysis, the results were submitted to ANOVA at $5 \%$ of the level of significance. The
\end{abstract}


results (mean \pm standard deviation) were: $30.14 \pm 1.34$ and $28.93 \pm 1.69 \mathrm{~cm}$ for scrotal circumference, $5.5 \pm 0.0$ and $4.64 \pm 0.8 \mathrm{ml}$ for volume of $2.71 \pm 0.49$ and $2.57 \pm 0.53 \%$ for swirling, $54.28 \pm 6.07$ and $67.86 \pm 9.51 \%$ for motility, $3.57 \pm 0.79$ and $3,28 \pm 0.75 \%$ for vigor, $657.14 \pm$ 97.59 and $678.57 \pm 138.01 \%$ for concentration, $7.57 \pm 1.27$ and $5.71 \pm 2.43 \%$ for total major defects, $5.43 \pm 2.76$ and $2.29 \pm 1.60 \%$ for total lower defects for young and adult bulls, respectively. The results observed in this study are within acceptable parameters for bovines of these age groups.

Keywords: bovine; Sperm concentration; Motility; semen; volume

\section{INTRODUÇÃO}

Muitas são as raças de bovinos registradas, sendo grande parte para produção de carne (BARBOSA, 1990). Segundo Barbosa (2000), com base no dicionário organizado por Mason (1988), há aproximadamente mil raças de bovinos no mundo, das quais 250 têm expressiva criação. No Brasil, há mais de 60 raças de bovinos que podem ser exploradas para produção comercial (BARBOSA, 1990; BARBOSA 2003). A raça bovina para produção de carne mais comum no país é o Nelore.

A carga genética Bos taurus do Blonde e a Bos indicus do Nelore, se complementaram, resultando em um produto promissor. Sinônimo de rusticidade, a raça possui extrema padronização, de forma quase cilíndrica e traseiro bem musculoso, com dorso e lombo de grande espessura e elevado comprimento corporal, estruturado em ossos finos porém fortes, características que Ihes conferem excepcionais rendimentos de carcaça (AGRONinformações, 2014).

A mais nova raça de corte criada e reconhecida no Brasil pelo Ministério da Agricultura, Pecuária e Abastecimento (MAPA) é a Blonel, oriunda do cruzamento de animais das raças Blonde e Nelore (ABBlonel, 2003). A raça Blonde, de origem francesa, tem um dos mais importantes programas de genética seletiva do mundo. Sua seleção genética teve atenção especial à precocidade sexual, fertilidade, habilidade materna e produção de carne de qualidade. Por outro lado, a raça Nelore, tradicional na produção de carne brasileira, traz a rusticidade e adaptação às condições geográficas e climáticas (ABBlonel, 2005).

O processo científico, que já completou mais de uma década, de sucessivos cruzamentos entre as duas raças-base, por meio da biotecnologia dos tempos modernos aplicada à pecuária, sempre com a utilização de sêmen de touros provados em testes de progênie, fixou o Blonel no grau sanguíneo de 5/8 Blonde com 3/8 Nelore (AGRONinformações, 2014).

Segundo Malmegrin (2006), os touros blonde utilizados na formação da raça Blonel passaram por teste de progênie, que tem como proposito verificar se o animal tem capacidade de transmitir suas características positivas para seus descendentes. $O$ cruzamento entre as duas raças originou um bovino rústico, musculoso, com bom ganho de peso, precocidade sexual, facilidade de parto e boa habilidade materna, de acordo com Malmegrin (2006).

Segundo Malmegrim (2006), a característica de rusticidade é essencial nas condições da pecuária brasileira. $\mathrm{O}$ touro Blonel apresenta a mesma boa performance de cobertura de fêmeas que o Nelore. Como os zebuínos, o Blonel possui pele escura e pelos curtos, de cor clara, que refletem os raios solares, permitindo-os andar longas distâncias em busca de vacas no estro.

Segundo um projeto apresentado ao Ministério da Agricultura Pecuária, a produção de novilhos criados a pasto, durante 31 meses, custou 22,51 reais por arroba, cotada na época a 50 reais - um lucro de $120 \%$. (ABBlonel). De acordo com Malmegrim (2006), o animal criado somente a pasto e feno, já atinge peso para abate em dois anos. Isso permite ao produtor aumentar em $50 \%$ a quantidade de gado na propriedade. 
Assim como a raça, os estudos científicos desses animais tiveram início a pouco, e muitos ainda nem foram publicados. A Embrapa, em São Carlos, SP, realizou um experimento com 18 animais de várias raças, com dieta de $13 \%$ de proteína bruta e $70 \%$ de nutrientes digestíveis totais. Foi observado peso inicial de $275 \mathrm{~kg}$ para animais cruzados Blonde/Nelore e peso final (após 69 dias de confinamento) de $405 \mathrm{~kg}$ (15,8 arrobas), ou seja, um ganho diário de 1,9 kg. No abate, o rendimento observado foi de 58,5\%. As outras raças presentes foram o Canchim, o Canchim/Nelore e o Limousin/Nelore (ABBlonel, 2006).

Em Ilha Solteira, SP, na Universidade Estadual "Júlio de Mesquita Filho", foi realizado um experimento onde o peso inicial de animais 3/8 Nelore e 5/8 Blonde (Blonel) era de 405,7 kg. O peso final foi de $511,1 \mathrm{~kg}$, com ganho diário de $1,35 \mathrm{~kg}$ e taxa de conversão de $8,3 \mathrm{~kg} \mathrm{MS} / \mathrm{kg} \mathrm{PV}$. A carcaça pesou 285,6 kg (19,026 arrobas), com rendimento de 55,85\% (ABBlonel, 2006).

Já em confinamento orientado pelo IZ de Sertãozinho, SP, na Fazenda Bethânia, o peso vivo inicial de animais castrados era $405 \mathrm{~kg}$, terminando com $535 \mathrm{~kg}$, com um ganho de 1,16 kg/dia em dieta de $12 \%$ de proteína bruta e $66 \%$ de NDT. Os animais inteiros, entre $20-24$ meses, iniciaram com $347 \mathrm{~kg}$, terminando em $509 \mathrm{~kg}$, com ganho diário de 1,572 kg (ABBlonel, 2006).

Em testagem comparativa com a raça Nelore, que foi monitorada pela Universidade de Illinois (EUA), em extremas condições de rusticidade na Chapada dos Veadeiros (GO), os bezerros filhos de Blonel sobre as vacas zebuínas, obtiveram o primeiro lugar no peso à desmama, tanto de macho como de fêmea, superando em $22 \%$ a média geral (ABBlonel, 2006).

Como apontado pelos resultados anteriores, a raça Blonel mostra-se muito promissora, principalmente devido a sua alta produção de carne e precocidade. No entanto, estes animais ainda não foram estudados quanto ao seu desempenho reprodutivo. Neste sentido, objetivou-se estudar a performance reprodutiva de touros da raça Blonel, ao exame andrológico, especificamente, os parâmetros de exame clínico e análise do ejaculado.

\section{MATERIAL E MÉTODOS}

Este estudo foi dispensado de avaliação pelo Comitê de Ética no Uso de Animais (CEUA). Foi realizado um levantamento de dados reprodutivos de um rebanho bovino da Fazenda Três Furnas, em Buriti Alegre - GO, referentes ao ano de 2012. Para este levantamento, foram estudados 14 touros da raça Blonel, os quais foram submetidos a exame andrológico entre 24 e 63 meses de idade naquele ano. Os animais foram classificados conforme a idade, sendo jovens ( 24 a 33 meses) e adultos (53 a 63 meses). O exame andrológico foi conduzido pelo mesmo médico veterinário, durante os meses de agosto e setembro, sob os mesmos padrões de manejo e alimentação.

O exame andrológico foi classificado por meio de três etapas diferentes. Primeiramente, a identificação do nome do animal, raça, idade, número de registro, proprietário e local do estudo. O segundo procedimento foi o exame clínico constituído do histórico do animal, exame clínico geral, exame clínico dos genitais (pênis, prepúcio, glândulas anexas e testículos), circunferência escrotal e aprumos. O terceiro e último foi avaliação do sêmen de todos os animais. Não foi avaliado o comportamento sexual dos animais.

A coleta de sêmen para análise foi conduzida pelo método de eletroejaculação, de acordo com as normas vigentes (segundo o Manual de Andrologia do Colégio Brasileiro de Reprodução Animal - CBRA). Imediatamente após a coleta, foram conduzidas as análises imediatas: volume do ejaculado, turbilhonamento, motilidade, vigor e concentração espermática. Foi observado se havia presença de outros tipos celulares (anormais) no ejaculado e registrado. A partir de uma gota de sêmen, fez-se um esfregaço em lâmina de microscopia.

Posteriormente, em laboratório, esta lâmina foi corada (coloração eosina-nigrosina) para a condução da análise mediata das células espermáticas (morfologia espermática) sob microscopia, onde foram avaliados todos os tipos de defeitos e suas freqüências. 
Os resultados dos exames andrológicos foram digitados em planilha e submetidos à análise e cálculo de médias. Para a análise estatística, os resultados médios (circunferência escrotal, volume do ejaculado, turbilhonamento, motilidade, vigor, concentração, total de defeitos maiores e menores) de touros jovens e adultos foram submetidos à Análise de Variância, ao nível de significância de 5\% (SAS, 2012).

\section{RESULTADOS E DISCUSSÃO}

Os resultados observados ao exame andrológico estão representados nas Tabelas 1 e 2 . Estes estão dentro dos parâmetros aceitáveis para bovinos de mesmas faixas etárias, de acordo com o preconizado pelo Colégio Brasileiro de Reprodução Animal. Não foi observada diferença significativa entre touros Blonel jovens e adultos para circunferência escrotal, volume do ejaculado, turbilhonamento, motilidade, vigor, concentração, total de defeitos maiores e total de defeitos menores $(p>0,05)$.

Tabela 1. Estimativas da média da circunferência escrotal e parâmetros seminais de touros da raça Blonel (média \pm desvio padrão).

\begin{tabular}{ccccccc}
\hline Idade & $\begin{array}{c}\text { Circunferênci } \\
\text { a escrotal } \\
(\mathbf{c m})\end{array}$ & $\begin{array}{c}\text { Volume } \\
\text { do } \\
\text { ejaculado } \\
(\mathbf{m l})\end{array}$ & $\begin{array}{c}\text { Turbilhonament } \\
\mathbf{0}(\%)\end{array}$ & $\begin{array}{c}\text { Motilidade } \\
\mathbf{( \% )}\end{array}$ & $\begin{array}{c}\text { Vigor } \\
\mathbf{( \% )}\end{array}$ & $\begin{array}{c}\text { Concentração } \\
(\mathbf{x 1 0} / \mathbf{m l})\end{array}$ \\
\hline Jovem & $30,14 \pm 1,34$ & $\begin{array}{c}5,50 \pm \\
0,00\end{array}$ & $2,71 \pm 0,49$ & $54,28 \pm 6,07$ & $3,57 \pm 0,79$ & $657,14 \pm 97,59$ \\
\hline Adulto & $28,93 \pm 1,69$ & $\begin{array}{c}4,64 \pm \\
0,80\end{array}$ & $2,57 \pm 0,53$ & $67,86 \pm 9,51$ & $3,28 \pm 0,75$ & $\begin{array}{c}678,57 \pm \\
138,01\end{array}$ \\
\hline
\end{tabular}

As médias de circunferência escrotal observadas neste estudo são relativamente semelhantes quanto às descritas em touros da raça Guzerá com idade a partir de 24 meses, que apresentaram o valor médio da circunferência escrotal de 30,95 \pm 1,17 cm (MAClEL et al., 2015). Já o volume é inferior ao descrito por Lucio et al. (2014). Mas o turbilhonamento é elevado quando comparado ao da raça Bosmara de 1,82 $\pm 1,83 \%$, segundo Dágola et al. (2013). Quanto ao vigor e à motilidade observados para o Blonel, são favoráveis àqueles descritos para a raça Nelore, cujo vigor é de 2,6 \pm 0,6 \% e a motilidade é 49,5 \pm 13,6 \% (DIAS et al., 2009). E a concentração espermática do Blonel é superior à observada por Maciel et al. (2015) para a raça Holandês $2,2 \mathrm{x}$ $10^{7} \pm 0,6 \times 10^{7} / \mathrm{ml}$.

Tabela 2. Total de defeitos dos espermatozoides de touros da raça Blonel (média \pm desvio padrão).

\begin{tabular}{ccc}
\hline Idade & Total de defeitos maiores (\%) & Total de defeitos menores (\%) \\
\hline Jovem & $7,57 \pm 1,27$ & $5,43 \pm 2,76$ \\
Adulto & $5,71 \pm 2,43$ & $2,29 \pm 1,60$ \\
\hline
\end{tabular}

Os touros da raça Blonel de ambas as faixas etárias apresentaram baixo índice de defeitos dos espermatozoides. Menor inclusive que o citado para a raça Bonsmara, onde seus defeitos maiores são de 4,25 $\pm 4,29 \%$ (DÁGOLA et al., 2013). Pode-se ainda ressaltar o baixo índice de defeitos dos touros da raça Blonel analisando o observado por Dias et al. (2009) para touros Nelore. Este é um ótimo indicativo, visto que a morfologia espermática é adotada como parte do controle de qualidade para decidir o destino do ejaculado. Alterações morfológicas dos espermatozoides estão associadas com baixa fertilidade em touros (ARRUDA et al., 2015).

Segundo o manual para exame andrológico e avaliação do sêmen animal, é recomendado que ejaculados de touros tenham no mínimo $70 \%$ de células espermáticas normais, sendo esta a 
característica mais importante para a fertilidade do touro no rebanho. Defeitos maiores não devem ultrapassar 20\% e defeitos menores em 30\% (ARRUDA et al., 2015).

\section{CONCLUSÃO}

O exame andrológico de touros da raça Blonel indicou bom potencial reprodutivo, independentemente se jovens ou adultos. No entanto, mais estudos se fazem necessários, devido ao baixo número de repetições.

\section{REFERÊNCIAS}

ARRUDA, R. P. Revista Brasileira de Reprodução Animal, v. 39, n. 1, 2015.

BARBOSA, P. F. Estratégias de Utilização de Recursos Genéticos em Sistemas de Produção de Bovinos de Corte. Sistema de Produção da Embrapa Pecuária Sudeste, n. 2, jul, 2003.

BARBOSA, P. F. Papel dos cruzamentos entre raças de corte. In: III Simpósio Nacional de Melhoramento Animal, Sociedade Brasileira de Melhoramento Animal... Anais do III Simpósio Nacional de Melhoramento Animal, 2000, p. 124-129.

COLÉGIO BRASILEIRO DE REPRODUÇÃO ANIMAL - CBRA. Manual para exame andrológico e avaliação do sêmen animal. 2.ed. Belo Horizonte :1998. p49.

LUCIO, A. C. Exploratory analysis of diferences in sperm morphology in Nelore and Gir (Bos indicus) bulls. Trop. Anim. Health Prod., n. 46, p. 765-770, 2014.

MASON, I. L. 1988. A world dictionary of livestock breeds, types and varieties, $3^{a}$ ed.

Wallingford: CAB International. 348p.

DÁGOLA, D. M.; PEGORER, M. F.; SOUZA, F. A. A.; BARREIROS, T. R. R.; BLASCH, W.; PATELLI, T. H. C. Parâmetros Obtidos no Exame Andrológico de Touros da Raça Bonsmara. Biosci. J., Uberlândia, v. 29, n. 3, p. 690-695, May/June 2013.

DIAS, C. J.; ANDRADE, V. J.; MARTINS, J. A. M.; EMERICK, L. L.; GONÇALVES, P. E.M.; FILHO, V. R. V. Classificação Andrológica por Pontos (CAP) de Touros Nelore (Bos taurus indicus) de Dois e Três anos de idade, Criados sob pastejo. Ciência Animal Brasileira, v. 10, n. 4, p. 1094-1099, out./dez. 2009.

MACIEL, J. P. O.; SILVA, E. J.; NASCIMENTO, P. S.; GUIDO, S. I.; SANTOS FILHO, A. S; BARTOLOMEU, C. C. Avaliação de parâmetros andrológicos de touros das raças Holandesa e Guzerá submetidos ao Semiárido. Scientia Plena, v. 11, n. 04. 2015.

SAS Institute Inc., 2012. SAS/ETS ${ }^{\circledR}$ Software: Changes and Enhancements for Release 6.12. In: SAS Institute Inc., Cary, NC. 112.

MALMEGTIM, S. Por que ele deu certo. Revista Globo Rural, v. 252 p. 68-70, 2006. Disponível em:>http://www.blonel.org.br/noticias/com-voces-o-blonel-globo-rural-252.pdf>. Acesso em: 6 fev. 2017 